\title{
Increased Framingham Coronary Heart Disease Risk Score in Dementia Caregivers Relative to Non-Caregiving Controls
}

\author{
Roland von Känela ${ }^{a, b}$ Brent T. Mausbach ${ }^{b}$ Thomas L. Patterson ${ }^{b}$ \\ Joel E. Dimsdale ${ }^{b}$ Kirstin Aschbacher ${ }^{b}$ Paul J. Mills ${ }^{b}$ Michael G. Ziegler ${ }^{c}$ \\ Sonia Ancoli-Israel ${ }^{\text {b, d }}$ Igor Grant ${ }^{\text {b }}$ \\ ${ }^{a}$ Department of General Internal Medicine, Division of Psychosomatic Medicine, University Hospital, \\ Berne, Switzerland; Departments of ${ }^{\mathrm{b}}$ Psychiatry and ${ }^{\mathrm{C}}$ Medicine, University of California San Diego, and \\ 'Veterans Affairs San Diego Healthcare System, La Jolla, Calif., USA
}

\section{Key Words}

Caregiving $\cdot$ Coronary heart disease $\cdot$ Dementia $\cdot$

Framingham Risk Score

\begin{abstract}
Background: Elderly individuals who provide care to a spouse suffering from dementia bear an increased risk of coronary heart disease (CHD). Objective: To test the hypothesis that the Framingham CHD Risk Score would be higher in dementia caregivers relative to non-caregiving controls. Methods: We investigated 64 caregivers providing in-home care for their spouse with Alzheimer's disease and 41 gender-matched non-caregiving controls. All subjects (mean age $70 \pm 8$ years, $75 \%$ women, $93 \%$ Caucasian) had a negative history of CHD and cerebrovascular disease. The original Framingham CHD Risk Score was computed adding up categorical scores for age, blood lipids, blood pressure, diabetes, and smoking with adjustment made for sex. Results: The average CHD risk score was higher in caregivers than in controls even when co-varying for socioeconomic status, health habits, medication, and psychological distress (8.0 \pm 2.9 vs. $6.3 \pm 3.0$ points, $p=0.013$ ). The difference showed a medium effect size (Cohen's $d=0.57$ ). A relatively higher blood pressure in caregivers than in controls made the greatest
\end{abstract}

\section{KARGER}

() 2008 S. Karger AG, Basel

Fax +41613061234 E-Mail karger@karger.ch www.karger.com www.karger.com/ger contribution to this difference. The probability (area under the receiver operator curve) that a randomly selected caregiver had a greater $\mathrm{CHD}$ risk score than a randomly selected non-caregiver was $65.5 \%$. Conclusions: Based on the Framingham CHD Risk Score, the potential to develop overt CHD in the following 10 years was predicted to be greater in dementia caregivers than in non-caregiving controls. The magnitude of the difference in the CHD risk between caregivers and controls appears to be clinically relevant. Clinicians may want to monitor caregiving status as a routine part of standard evaluation of their elderly patients' cardiovascular risk.

Copyright $\odot 2008$ S. Karger AG, Basel

\section{Introduction}

The risk of overall mortality and incident coronary heart disease (CHD) has been shown to be increased in community-dwelling elderly experiencing strain in providing care to their disabled spouse $[1,2]$. Compared with other settings of family caregiving, providing care to a demented spouse is particularly demanding [3]. Dementia caregiving has been reported to take a toll on caregivers' mental and physical health [4], with changes in car- 
diovascular health being particularly noteworthy. Thus, spousal Alzheimer caregivers are reported to show accelerated development of systemic hypertension [5] and greater prevalence of $\mathrm{CHD}$ [6] compared to non-caregivers. In addition, psychological distress associated with the burden of caregiving was noted to reduce the time to cardiovascular disease manifestations in dementia caregivers [7].

A variety of behavioral and pathophysiological mechanisms may lead from the perceived chronic stress of caregiving directly or via neuroendocrine and sympatho-adrenal-medullary changes downstream to a high cardiovascular risk profile and eventually CHD $[8,9]$. For instance, caregiving strain has been associated with elevated blood pressure [10], dyslipidemia [11], hyperglycemia and hyperinsulinemia [12], as well as low physical activity [13]. Additional atherosclerotic risk factors related to coagulability and inflammation have also been shown to cluster in dementia caregivers [14].

Because different cardiovascular risk factors tend to aggregate in the same individual, epidemiologists have proposed estimating a person's cardiovascular risk based on a scoring system that combines several risk factors [15]. For instance, the Framingham CHD Risk Score is a simple algorithm to predict CHD over a 10 -year interval in a predominantly Caucasian population of men and women without known CHD at baseline [16]. The Framingham score considers categorical variables for age, blood lipids, blood pressure, diabetes, and smoking. The cardiovascular risk of caregivers has not previously been estimated by any operationalized algorithm. The availability of such a systematic evaluation of CHD risk might help identify dementia caregivers at risk of premature manifestation of angina pectoris, myocardial infarction, and CHD death [16].

The risk that a caregiver will die within 1 year of hospitalizing a spouse for dementia is increased in both female and male partners [17]. Also, the cardiovascular biology of caregivers is affected by care placement or bereavement after the death of the demented spouse $[10,18]$. To preclude dilution of the CHD risk score by physical effects of transitions in the caregivers' and patients' home situation, we assessed the risk score in caregivers still providing in-home care to their demented spouse. As a primary analysis, we determined the Framingham CHD Risk Score in a sample of Alzheimer caregivers and noncaregiving controls. Although the Framingham score makes no adjustment for variables which may clearly affect risk factor scores and CHD outcome [16], we performed a secondary analysis controlling for socioeco- nomic status, health behavior, psychological distress and medication. We hypothesized that, even when controlling for these variables, dementia caregivers would show a significantly higher $\mathrm{CHD}$ risk score than controls.

\section{Study Participants and Methods}

A total of 106 individuals with a complete data set and all free from clinical CHD and cerebrovascular disease as determined by a structured medical history were part of this study. Sixty-five participants were caregivers of a spouse with physician-diagnosed Alzheimer's disease and 41 were non-caregiving controls. All participants volunteered for a research study examining the effects of chronic stress on physical and mental health. They gave written informed consent to the study protocol, which was approved by the University of California at San Diego (UCSD) Institutional Review Board. Caregivers were recruited primarily from the University of California at San Diego Alzheimer's Disease Research Center, community support groups, and local senior centers and medical clinics. Non-caregiving controls with similar demographic characteristics were recruited primarily from local senior citizen health fairs and later through snowball referrals from both caregivers and non-caregivers already enrolled in the study.

Both caregivers and non-caregivers were required to be 55 years of age or older, currently married and living at home with their spouses, and in generally good mental and physical health. In addition, caregivers were required to be providing primary care for a spouse with a physician-based diagnosis of Alzheimer's disease. Exclusion criteria for all participants included receiving current treatment with anticoagulants and having severe hypertension (i.e. blood pressure $>200 / 120 \mathrm{~mm} \mathrm{Hg}$ ).

Measures

Coronary Heart Disease Risk Score

We calculated the original Framingham CHD Risk Score for both caregivers and non-caregivers according to the formula presented by Wilson et al. [16]. This formula assigns risk scores for each of six CHD risk categories, namely age, total cholesterol, high-density lipoprotein (HDL) cholesterol, blood pressure, diabetes status (yes vs. no), and current smoking status (yes vs. no). Total cholesterol and HDL cholesterol were assessed in serum by the Beckman-Coulter LX20 PRO. After 5 min of sitting, blood pressure was assessed in duplicate with an interval of 15 min using a Critikon Dinamap 8100 noninvasive blood pressure monitor. When systolic and diastolic blood pressure fell into different categories, the higher category defined classification for the blood pressure risk category. Participants were considered diabetic when casual glucose levels exceeded $150 \mathrm{mg} / \mathrm{dl}$. Plasma glucose was determined by the Beckman-Coulter LX20 PRO. Given differential rates of CHD for males and females, risk scores were adjusted by participant sex. Once scores from each of these risk categories were calculated, an overall CHD risk score was created by summing scores from each of the six risk categories. Higher total scores therefore indicate greater $\mathrm{CHD}$ risk or, more precisely, a relatively higher predictive value for the development of clinical $\mathrm{CHD}$ in the 10 years subsequent to the time of baseline assessment of the score [16]. 


\section{Demographic Characteristics}

In addition to age (in years) and sex, we employed the participant's years of formal education as a proxy for socioeconomic status. For this purpose, we used Hollinghead's [19] education categories that range from 1 (graduate level training) to 7 (less than 7 th grade education); thus, higher scores correspond to less education. The year when Alzheimer dementia was diagnosed in the spouse was obtained from the caregiver and verified by the research nurse by, e.g., reviewing medical records.

Health Behavior and Medication

A research nurse conducted a structured medical history asking about present health habits and medications. Participants rated their current level of the following health behaviors: (a) whether or not they currently smoke cigarettes; (b) the average number of reported hours of sleep per $24 \mathrm{~h}$; (c) the number of days/week playing sports, and (d) number of days/week consuming alcoholic beverages. Participants were also categorized as to whether they were currently taking antihypertensives or statins.

\section{Psychological Distress}

Participants completed a global self-report measure of stress asking to which extent they felt overwhelmed or overloaded by life responsibilities [20]. The four items capture feelings of exhaustion (i.e. 'you are exhausted when you go to bed at night'), time constraints (i.e. 'you have more things to do than you can handle'; 'you don't have time just for yourself'), and frustration (i.e. 'you work hard but never seem to make any progress'). Responses are rated on a 4 -point Likert scale ranging from $1=$ 'not at all' to $4=$ 'completely'. Items are summed to create an overall stress score (range 4-16).

Depressive symptoms were assessed using the 17-item Hamilton Rating Scale for Depression [21]. A research nurse provided ratings on a 0 - to 4 - or 0 - to 2-point scale on each of the 17 items, with higher scores indicating greater depressive symptoms. The Hamilton Rating Scale for Depression is a brief yet comprehensive measure of depressive symptoms, with well-documented reliability and validity $[21,22]$.

\section{Statistical Analyses}

Data were analyzed using the SPSS (13.0) statistical software package (Chicago, Ill., USA) and testing was two-tailed with the significance level set at $p<0.05$. All variables were examined for deviations from normality by the Kolmogorov-Smirnov test. Before analyses, we obtained a normal distribution of education level, glucose level, health behavior variables, psychological variables, years since the diagnosis of Alzheimer's disease, and individual risk scores of the six CHD risk categories by normal score transformation according to Blom [23]. For clarity, we provide non-transformed means \pm SD throughout. Use of antihypertensive drugs and statins was coded with a dummy variable (no medication $=0$, confirmed use of medication $=1$ ).

We used independent-samples t tests and $\chi^{2}$ tests to investigate group differences in continuous and categorical measures, respectively. Pearson's correlation analysis was used to estimate the univariate relationship between two variables. Univariate analysis of covariance was applied to test for a difference in the $\mathrm{CHD}$ risk score between caregivers and controls adjusted for different blocks of covariates. In order to estimate the effect size of this difference, we calculated Cohen's d [24] and the area un- der the receiver operator curve (AUC) [25]. In this case the AUC represents the probability that a randomly selected caregiver has a greater CHD risk score than a randomly selected non-caregiver.

\section{Results}

\section{Subject Characteristics}

The 106 participants were on average $70.2 \pm 8.4$ years old, predominantly women (75\%), and of Caucasian ethnicity (93\%). Table 1 gives the comparison of characteristics between the 65 dementia caregivers and the 41 noncaregiving controls. Caregivers were older and had higher systolic blood pressure than their non-caregiving counterparts. Socioeconomic status, health habits and medication use did not differ between groups. As expected, depressive symptom levels and role overload scores were higher in caregivers than in controls, although the majority of the participants did not meet clinical criteria for major depression.

\section{Coronary Heart Disease Risk Score}

Bivariate Associations

Caregivers had a significantly higher Framingham CHD Risk Score than non-caregiving controls (7.95 \pm 2.94 vs. $6.34 \pm 2.66, \mathrm{p}=0.005)$. Cohen's $\mathrm{d}$ for this difference was 0.57 , suggesting a medium effect size. The probability that a randomly selected caregiver had a greater $\mathrm{CHD}$ risk score than a randomly selected non-caregiver (AUC) was 65.5\%. Time since the diagnosis of Alzheimer dementia averaged $4.0 \pm 3.1$ (range 0.5-12) years. Although pointing in the expected direction, the positive correlation between the number of years since the diagnosis of dementia in the spouse and the CHD risk score of caregivers did not reach statistical significance $(r=$ $0.18 ; \mathrm{p}=0.15)$.

Among all participants, a higher Framingham CHD Risk Score was associated with a lower education level ( $r=0.22, p=0.024)$ and fewer days per week that the subjects drank alcoholic beverages $(r=-0.33, p=0.001)$. The bivariate relationships between the risk score and hours of sleep $(p=0.50)$, sports days $(p=0.75)$, depressive symptoms $(\mathrm{p}=0.26)$, and role overload $(\mathrm{p}=0.32)$ were not significant. Also, the CHD risk score was similar in subjects who took antihypertensive medication $(\mathrm{p}=0.18)$ or statins $(p=0.13)$ compared to those who were not on these medications. Systolic $(p=0.12)$ and diastolic $(p=0.51)$ blood pressure were not significantly different between users and non-users of antihypertensive medication. Statin users had significantly lower HDL cholesterol (46.1 \pm 11.3 
Table 1. Subjects' characteristics

\begin{tabular}{lccc}
\hline Variable & Caregivers $(\mathrm{n}=65)$ & Controls $(\mathrm{n}=41)$ & $\mathrm{p}$ value \\
\hline Male/female, \% & $28 / 72$ & $22 / 78$ & 0.509 \\
Caucasian/other, \% & $91 / 9$ & $98 / 2$ & 0.244 \\
Education level, score & $2.5 \pm 1.1$ & $2.4 \pm 1.2$ & 0.810 \\
Age, years & $72.7 \pm 8.6$ & $67.5 \pm 6.7$ & 0.001 \\
Total cholesterol, mg/dl & $200.6 \pm 35.7$ & $194.4 \pm 36.7$ & 0.395 \\
HDL cholesterol, mg/dl & $55.4 \pm 16.5$ & $53.9 \pm 13.0$ & 0.654 \\
Systolic blood pressure, mm Hg & $132.3 \pm 16.0$ & $119.9 \pm 12.3$ & $<0.001$ \\
Diastolic blood pressure, mm Hg & $64.8 \pm 10.0$ & $62.3 \pm 10.4$ & 0.230 \\
Casual blood glucose, mg/dl & $102.5 \pm 30.4$ & $98.5 \pm 21.8$ & 0.753 \\
Current smokers, \% & 3 & 7 & 0.373 \\
Hours of sleep/24 h & $6.9 \pm 1.2$ & $7.0 \pm 1.1$ & 0.831 \\
Sports days/week & $0.7 \pm 1.6$ & $0.9 \pm 1.9$ & 0.586 \\
Alcohol days/week & $2.7 \pm 3.0$ & $3.2 \pm 2.7$ & 0.146 \\
Antihypertensive medication, \% & 40 & 27 & 0.166 \\
Statin use, \% & 18 & 24 & 0.464 \\
Depressive symptoms, score & $4.4 \pm 4.0$ & $2.3 \pm 2.1$ & 0.003 \\
Role overload, score & $10.8 \pm 11.5$ & $6.1 \pm 1.9$ & $<0.001$ \\
\hline
\end{tabular}

Values are means $\pm \mathrm{SD} . \mathrm{HDL}=$ High-density lipoprotein.

Table 2. Coronary heart disease risk score between caregivers and controls

\begin{tabular}{llll}
\hline Covariates entered & Caregivers $(\mathrm{n}=65)$ & Controls $(\mathrm{n}=41)$ & $\mathrm{p}$ value \\
\hline Block 1: education level & $7.94 \pm 2.78(7.26-8.63)$ & $6.30 \pm 2.78(5.50-7.22)$ & 0.005 \\
Block 2: hours of sleep/24 h, sports days/week, alcohol days/week & $7.86 \pm 2.70(7.20-8.53)$ & $6.49 \pm 2.71(5.65-7.33)$ & 0.013 \\
Block 3: antihypertensive medication, statin use & $7.84 \pm 2.68(7.18-8.50)$ & $6.52 \pm 2.70(5.69-7.36)$ & 0.017 \\
Block 4: depressive symptoms, role overload & $7.97 \pm 2.90(7.25-8.68)$ & $6.32 \pm 3.03(5.38-7.26)$ & 0.013 \\
\hline
\end{tabular}

Analyses of covariance; values are means \pm SD (95\% confidence interval). Blocks $1-4$ of covariates were subsequently entered into the equation.

vs. $57.0 \pm 15.4 \mathrm{mg} / \mathrm{dl}, \mathrm{p}=0.002)$ and also marginally lower total cholesterol $(186.0 \pm 42.7$ vs. $201.4 \pm 33.7 \mathrm{mg} / \mathrm{dl}$, $\mathrm{p}=0.074)$ than participants not taking statins.

In order to test for hidden socioeconomic, medical, and psychological factors potentially related to age, we computed a series of additional bivariate correlation analyses with age as the independent variable across the entire sample of caregivers and controls. Age showed no significant associations (all $\mathrm{p}>0.05$ ) with education, cardiovascular risk factors (i.e. lipids, blood pressure, glucose, smoking), medication (i.e. use of antihypertensives and statins), health behavior (i.e. hours of sleep, sports days, days per week subjects drank alcoholic beverages), depressive symptoms, and role overload (data not shown in detail).
Analysis of Covariance

Table 2 shows the results of the univariate analysis of covariance for the difference in the CHD risk score between caregivers and controls considering four blocks of covariates (i.e. socioeconomic status, health behavior, medication use, and psychological distress). Caregivers had a significantly higher CHD risk score than controls independent of all covariates. In the last model, observed power for caregiver status was 0.71 with $\alpha=0.05$, and caregiver status explained a unique $6.3 \%$ of the variance in the $\mathrm{CHD}$ risk score. Effect size for the difference in the $\mathrm{CHD}$ risk score between caregivers and controls was also not affected after controlling for covariates (Cohen's $\mathrm{d}=$ 0.57). Similarly, the likelihood that caregivers had higher CHD risk scores than non-caregivers (AUC) was 65.5\%. 
Table 3. Scores of individual risk factors between caregivers and controls

\begin{tabular}{lccr}
\hline Scores & Caregivers $(\mathrm{n}=65)$ & Controls $(\mathrm{n}=41)$ & p value \\
\hline Age & $7.54 \pm 0.66(5-8)$ & $7.46 \pm 1.03(4-8)$ & 0.911 \\
Total cholesterol & $0.31 \pm 1.18(-3$ to 2$)$ & $0.15 \pm 1.15(-3$ to 3$)$ & 0.313 \\
HDL cholesterol & $-0.34 \pm 2.19(-3$ to 5$)$ & $-0.34 \pm 1.88(-3$ to 2$)$ & 0.712 \\
Blood pressure & $0.20 \pm 1.80(-3$ to 3$)$ & $-1.12 \pm 1.75(-3$ to 2$)$ & $<0.001$ \\
Glucose & $0.18 \pm 0.58(0-2)$ & $0.04 \pm 0.31(0-2)$ & 0.123 \\
Smoking & $0.06 \pm 0.35(0-2)$ & $0.15 \pm 0.53(0-2)$ & 0.365 \\
\hline
\end{tabular}

Values are means $\pm \mathrm{SD}$ (range). $\mathrm{HDL}=$ High-density lipoprotein.

In addition to caregiver status, a lower education level $(\mathrm{p}=0.009)$ and fewer alcohol days per week $(\mathrm{p}=0.008)$ were also significant covariates of higher CHD risk score, uniquely explaining 6.9 and $7.1 \%$ of the variance, respectively, in the final model. In contrast, reported hours of sleep per $24 \mathrm{~h}(\mathrm{p}=0.79)$, sports days per week $(\mathrm{p}=0.66)$, use of antihypertensives $(\mathrm{p}=0.12)$ and of statins $(\mathrm{p}=$ $0.15)$, as well as depressive symptoms $(\mathrm{p}=0.82)$, and role overload $(p=0.29)$ were not significant covariates of the $\mathrm{CHD}$ risk score in the final model.

\section{Post hoc Analyses}

In post hoc analyses, we aimed to identify which of the individual categorical scores for age, blood pressure, lipids, diabetes, and smoking would contribute most to the difference in the total CHD risk score between caregivers and controls. Table 3 demonstrates the comparison of the individual risk factor scores between groups. Notably, caregivers had a higher blood pressure score than controls $(\mathrm{p}<0.001)$, whereas all other risk factor scores were not significantly different between groups. In particular, although caregivers were on average significantly older than non-caregivers (table 1), age was not an evident contributor to the relatively higher CHD risk score in caregivers ( $p>0.90)$. Specifically, although caregivers were significantly older than controls (table 1 ), the contribution of the age score to the overall CHD risk score was similar for the two groups.

\section{Discussion}

We found that elderly community-dwelling spousal caregivers of Alzheimer's disease patients had a significantly higher Framingham CHD Risk Score than noncaregiving controls. These data suggest that dementia caregivers may be at a higher risk of developing CHD (i.e. angina pectoris, myocardial infarction, or death from CHD) over the next 10 years relative to controls. This finding, along with the observation that the blood pressure risk score was also higher in caregivers than in controls, substantiates accumulating evidence for compromised cardiovascular health in dementia caregivers [57]. Notably, of the individual risk scores, the blood pressure score alone was higher in caregivers, even though $40 \%$ of caregivers took antihypertensive medications, compared with $27 \%$ of controls ( $p$ NS). This finding concurs with previous studies showing more rapid development of hypertension over time in Alzheimer caregivers compared to non-caregiving controls [5] and increased blood pressure in diverse populations of chronically stressed individuals [26].

The effect size of the difference in the CHD risk score between caregivers and controls was medium, suggesting it could be of clinical relevance. By estimating the AUC, we report a $66 \%$ likelihood that caregivers will have a higher CHD risk score than non-caregivers. Since the Framingham score is easily calculated from information routinely assessed in physician's offices, we propose that the Framingham score may be a useful adjunct for clinicians wishing to estimate the added CHD risk of their elderly caregiving patients.

Our data suggest that clinicians may want to monitor caregiving status as a routine part of standard patient evaluation. In this way, the physician can work with the caregiving patient to identify resources that lessen caregiving burden, including sources of practical and emotional support, and individualized strategies aimed at increasing mastery, reducing stress, and thereby enhancing well-being [27]. Moreover, obvious cardiovascular risk factors should be treated in caregivers. Particularly, the higher blood pressure observed in our caregivers relative to the non-caregiving controls might easily be lowered by antihypertensive medication as has been shown in the 
elderly in general [28]. Numerous prospective trials have demonstrated that blood pressure control decreases cardiovascular risk in the elderly [28]; future studies may explore whether such interventions could be of particular benefit in terms of reducing CHD risk in dementia caregivers. The original Framingham score does not consider control variables. Even when we controlled for socioeconomic status, health habits, psychological stress, and medication, the predictive value of caregiver status for the CHD risk score was maintained. Although other studies of caregivers [7] and other groups [29] have linked depression to CHD risk, our measures of psychological distress did not account for substantial amounts of the $\mathrm{CHD}$ risk score. It is possible that because the overall level of depressive symptoms was subclinical in our sample the restricted range in this variable limited statistical power to find true effects.

The mechanisms linking caregiving to increased cardiovascular risk are unknown, but have been thought to involve at least the upregulation of the sympathetic-adrenal-medullary system and hypothalamic-pituitary-adrenal axis [9]. For instance, and as seen in other models of chronic stress, sympathetic overactivity in dementia caregivers might increase blood pressure in the long run $[5,30]$. Also, an increase in peripheral cortisol with chronic stress could give rise to an increase in blood glucose levels and the metabolic syndrome, respectively [6, 31].

There are two limitations to our study. First, some authors feel that the clinical value of risk scoring for CHD, including the Framingham scores, is questionable [32, 33], while others advocate that risk scores may well have their value but should be refined [34]. Our study is a first step in applying such risk score techniques specifically to dementia caregivers and to test whether by this approach
CHD risk could reasonably be monitored in this population. We therefore selected the Framingham Risk Score because it is a widely known tool and may have merits in a predominantly Caucasian population such as our study participants [16]. Future studies should assess the Framingham score along with more refined cardiovascular risk scores [34] in order to replicate our finding. Even more importantly, such studies should compare the CHD risk obtained by different scoring systems in the same caregiver population both cross-sectionally and prospectively. Second, our subjects were in comparatively good health. For instance, only a few subjects were smokers and all were able to provide in-home care in spite of advanced age, suggesting that few caregivers were very frail. Our findings might therefore not be generalized to dementia caregivers who are physically and mentally less fit.

In summary, we found that elderly individuals free of clinically evident CHD and who had to provide in-home care to their demented spouse had an elevated CHD risk score, particularly due to the contribution of increased blood pressure. Based on the Framingham score, dementia caregivers had a potentially increased risk of developing overt $\mathrm{CHD}$ in the next 10 years. The finding supports the notion that physical impairment with caregiving strain may particularly target the cardiovascular system and is clinically relevant.

\section{Acknowledgments}

The authors wish to thank Susan Calleran, MA, Carolyn Swenerton, RN, and Sharyn Wilensky, RN, for their assistance in the conduct of this study. Primary support for this study derived from AG 15301 from the National Institute on Aging (NIA). Added support was from NIA award AG 23989.

\section{References}

1 Schulz R, Belle SH, Czaja SJ, McGinnis KA, Stevens A, Zhang S: Long-term care placement of dementia patients and caregiver health and well-being. JAMA 2004;292:961967.

-2 Lee S, Colditz GA, Berkman LF, Kawachi I: Caregiving and risk of coronary heart disease in US women: a prospective study. Am J Prev Med 2003;24:113-119.

- 3 Ory MG, Hoffman RR 3rd, Yee JL, Tennstedt S, Schulz R: Prevalence and impact of caregiving: a detailed comparison between dementia and nondementia caregivers. Gerontologist 1999;39:177-185

\footnotetext{
4 Schulz R, O’Brien AT, Bookwala J, Fleissner K: Psychiatric and physical morbidity effects of dementia caregiving: prevalence, correlates, and causes. Gerontologist 1995;35: 771-791.

-5 Shaw WS, Patterson TL, Ziegler MG, Dimsdale JE, Semple SJ, Grant I: Accelerated risk of hypertensive blood pressure recordings among Alzheimer caregivers. J Psychosom Res 1999;46:215-227.

6 Vitaliano PP, Scanlan JM, Zhang J, Savage MV, Hirsch IB, Siegler IC: A path model of chronic stress, the metabolic syndrome, and coronary heart disease. Psychosom Med 2002;64:418-435.
}

-7 Mausbach BT, Patterson TL, Rabinowitz Y, Grant I, Schulz R: Depression and distress predict time to cardiovascular disease in dementia caregivers. Health Psychol 2007;26: 539-544

8 Rozanski A, Blumenthal JA, Davidson KW, Saab PG, Kubzansky L: The epidemiology, pathophysiology, and management of psychosocial risk factors in cardiac practice: the emerging field of behavioral cardiology. J Am Coll Cardiol 2005;45:637-651.

-9 Grant I: Caregiving may be hazardous to your health. Psychosom Med 1999;61:420423. 
10 Grant I, Adler KA, Patterson TL, Dimsdale JE, Ziegler MG, Irwin MR: Health consequences of Alzheimer's caregiving transitions: effects of placement and bereavement. Psychosom Med 2002;64:477-486.

-11 Vitaliano PP, Russo J, Niaura R: Plasma lipids and their relationships with psychosocial factors in older adults. J Gerontol B Psychol Sci Soc Sci 1995;50:P18-P24.

-12 Vitaliano PP, Scanlan JM, Krenz C, Fujimoto W: Insulin and glucose: relationships with hassles, anger, and hostility in nondiabetic older adults. Psychosom Med 1996;58:489499.

-13 Schulz R, Newsom J, Mittelmark M, Burton L, Hirsch C, Jackson S: Health effects of caregiving: the caregiver health effects study: an ancillary study of the Cardiovascular Health Study. Ann Behav Med 1997;19:110-116.

14 von Känel R, Dimsdale JE, Mills PJ, et al: Effect of Alzheimer caregiving stress and age on frailty markers interleukin-6, $\mathrm{C}$ reactive protein, and D-dimer. J Gerontol A Biol Sci Med Sci 2006;61:963-969.

15 Wilson PW: Estimating the risk for atherothrombosis: are current approaches sufficient? Eur J Cardiovasc Prev Rehabil 2005; 12:427-432.

-16 Wilson PW, D’Agostino RB, Levy D, Belanger AM, Silbershatz A, Kannel WB: Prediction of coronary heart disease using risk factor categories. Circulation 1998;97:18371847.
17 Christakis NA, Allison PD: Mortality after the hospitalization of a spouse. N Engl J Med 2006;354:719-730.

18 Mausbach BT, Aschbacher K, Patterson TL, et al: Effects of placement and bereavement on psychological well-being and cardiovascular risk in Alzheimer's caregivers: a longitudinal analysis. J Psychosom Res 2007;62: 439-445.

19 Hollingshead AB: Social Class and Mental Illness. New York, Wiley, 1958.

20 Pearlin LI, Mullan JT, Semple SJ, Skaff MM: Caregiving and the stress process: an overview of concepts and their measures. Gerontologist 1990;30:583-594.

21 Hamilton M: Standardised assessment and recording of depressive symptoms. Psychiatr Neurol Neurochir 1969;72:201-205.

22 Hamilton M: General problems of psychiatric rating scales (especially depression); in Pichot P, Olivier-Martin R (eds): Psychological Measurements in Psychopharmacology. Mod Trends Pharmacopsychiatry. Basel, Karger, 1974, vol 7, pp 125-138.

23 Blom G: Statistical Estimates and Transformed Beta-Variables. New York, Wiley, 1958.

24 Cohen J: Statistical Power Analysis for the Behavioral Sciences. Hillsdale, Erlbaum, 1988.

25 Kraemer HC, Morgan GA, Leech NL, Gliner JA, Vaske JJ, Harmon RJ: Measures of clinical significance. J Am Acad Child Adolesc Psychiatry 2003;42:1524-1529.

26 Pickering TG: Mental stress as a causal factor in the development of hypertension and cardiovascular disease. Curr Hypertens Rep 2001;3:249-254
27 Schulz R, Martire LM: Family caregiving of persons with dementia: prevalence, health effects, and support strategies. Am J Geriatr Psychiatry 2004;12:240-249.

28 Wing LM, Reid CM, Ryan P, Beilin LJ, Brown MA, Jennings GL, Johnston CI, McNeil JJ, Macdonald GJ, Marley JE, Morgan TO, West MJ: A comparison of outcomes with angiotensin-converting-enzyme inhibitors and diuretics for hypertension in the elderly. $\mathrm{N}$ Engl J Med 2003;348:583-592.

29 Barth J, Schumacher M, Herrmann-Lingen $\mathrm{C}$ : Depression as a risk factor for mortality in patients with coronary heart disease: a metaanalysis. Psychosom Med 2004;66:802-813.

30 Landsbergis PA, Schnall PL, Pickering TG, Warren K, Schwartz JE: Life-course exposure to job strain and ambulatory blood pressure in men. Am J Epidemiol 2003;157: 998-1006.

31 Peeke PM, Chrousos GP: Hypercortisolism and obesity. Ann NY Acad Sci 1995;771: 665-676.

32 Beswick A, Brindle P: Risk scoring in the assessment of cardiovascular risk. Curr Opin Lipidol 2006;17:375-386.

33 Brindle PM, Beswick AD, Fahey T, Ebrahim SB: The accuracy and impact of risk assessment in the primary prevention of cardiovascular disease: a systematic review. Heart 2006;92:1752-1759.

>34 Jurgensen JS: The value of risk scoring. Heart 2006;92:1713-1714. 